\title{
Use of Comparison Films for Breast Cancer Screening and Diagnosis Among Florida Radiologists
}

\author{
N Wadhwa, M Hernandez, D Steinkohl, A Bhasin, C Bailey, A Perez
}

\section{Citation}

N Wadhwa, M Hernandez, D Steinkohl, A Bhasin, C Bailey, A Perez. Use of Comparison Films for Breast Cancer Screening and Diagnosis Among Florida Radiologists. The Internet Journal of Radiology. 2009 Volume 12 Number 1.

\begin{abstract}
Background: In the United States, there are on average, 200,000 cases of breast cancer diagnosed each year. Research studies conducted by the National Cancer Institute have estimated that one out of every eight women in the United States (12.5\%) will develop breast cancer during her lifetime, with the highest percentage of women diagnosed between ages 40 and 50. Due to the good prognosis of early detection, much research has recently surfaced on the efficacy of diagnostic testing of cancerous breast tissue. However, research has shown that up to $4 \%$ of breast cancers are missed, and a major factor is the lack of comparison mammogram. Methods: A one page anonymous survey was sent to licensed Florida Radiologists using randomization provided by Medical Marketing Services, Inc (MMS). The number of radiologists registered within the MMS database in the state of Florida is 98 . A number of surveys were also sent to radiologists working within the Memorial Healthcare System of Broward County, FL. The total number of responses collected was 22. Results: According to the collected data, forty-one percent of Florida radiologists review mammograms in their practice. Twenty-three percent of radiologists review mammograms more than half the time, while eighteen percent review mammograms less than half the time. Of the Florida radiologists who review mammograms, ninety-five percent believe that comparing past mammograms is very important in making a correct diagnosis. However, twenty-two percent of those radiologists never compare past films. Twenty-four percent of Florida radiologists reading mammograms have been sued for malpractice at least one time. There is a strong inverse relationship between comparing mammograms and likelihood to be sued. Florida radiologists who think comparing mammograms is "very important" were less likely to be sued for malpractice $(R=0.99, p<0.0001)$. Conclusions: The objective of the study was to establish a relationship between mammogram comparison and misdiagnosis rates of breast cancer. The results show a strong inverse relationship, in that an increase of mammogram comparison will likely decrease the failure to diagnose breast cancer. Since radiologists agree that comparing mammograms is essential, comparison must be emphasized in continuing educational courses and must be made part of routine procedure. Moreover, primary care physicians must educate their patients as to the importance of having past mammograms in their possession so that present and future mammograms can be compared.
\end{abstract}

\section{BACKGROUND}

In the United States, there are on average, 200,000 cases of breast cancer diagnosed each year ${ }^{1}$. Abnormal growth of cells in the tissue of the breast, which multiply and divide at an uncontrolled rate, is characteristic of breast cancer. The most common type of breast cancer-ductal

carcinoma-begins in the lining of the ducts, which connects the nipples to other parts of the breast. These cancerous cells can invade nearby tissues, metastasize through the bloodstream and lymphatic system, and rapidly spread to other parts of the body.

Research studies conducted by the National Cancer Institute have estimated that one out of every eight women in the United States (12.5\%) will develop breast cancer during her lifetime, with the highest percentage of women diagnosed between ages 40 and $50^{2}$. According to Domchek SM and Weber BL, gene mutations are responsible for nearly all hereditary breast cancers, making breast cancer the second leading cause of female cancer deaths, killing $20 \%$ of women who develop the disease ${ }^{3}$. Sutter Health's Breast Cancer Project has shown that when caught in its earliest stages, most breast cancers confined to the breast ducts or breast tissue are curable. Women diagnosed during stage 0 or 1 of the cancer experience a 95 percent cure rate ${ }^{4}$. Hence, to help with early detection of cancerous breast tissue current recommendations include monthly self breast exams and a mammogram every one to two years after the age of 40. On the other hand, if a cancerous tissue of the breast goes undiagnosed, the rates of survival decrease 
considerably during stages II, III or IV of the cancer, as the cancerous cells have usually spread to surrounding lymph nodes ${ }^{4}$.

Due to the good prognosis of early detection, much research has recently surfaced on the efficacy of diagnostic testing of cancerous breast tissue. For example, studies evaluating the accuracy of needle biopsies, ultrasonograms, and breast mammograms have been extensively studied to detect when cancer cells begin invading nearby tissues. However, studies have not yet compared the value of tracking the progression of breast tissue by comparing previous mammograms to more current ones. According to malpractice data evaluated by (Gandhi, et. al), it is known that breast cancer is the most commonly misdiagnosed medical condition ${ }^{5}$. In their study conducted on malpractice claims and medical lawsuits, 59\% of the claims involved misdiagnosis, while claims involving breast cancer totaled $24 \%$. In $55 \%$ of the misdiagnosis cases, there was a failure to order an appropriate diagnostic test, while in $37 \%$ of the cases an error in the interpretation of diagnostic tests was made ${ }^{5}$. It was the conclusion of these authors that the misdiagnosis of medical conditions often results from an error in the interpretation of diagnostic tests. With the high rates of misdiagnosis and errors in interpretation, it is the hypothesis of the authors of this study that requiring all radiologists to reference previous diagnostic films will reduce the rates of failure to diagnose cancerous breast tissues.

While regular clinical and patient self-breast exams are important, mammography has still been found to be the best screening tool ${ }^{4}$. Mammography can detect cancers up to two years before palpation. Technological advances, such as digital mammography and computer-aided detection (CAD), have greatly improved mammography accuracy.

Moreover, the efficacy of screening by mammography has been shown in randomized controlled trials in women aged 50 years and older. In the research study by (Moss, et.al), the authors studied the mortality rates of women after inviting women to receive annual mammograms from age 40 . It was observed that after 10 years there was a reduction in breastcancer mortality in the intervention group ${ }^{6}$.

Since medical diagnostic tests are not perfect and can even miss detection of cancerous tissues, requiring radiologists to reference a patient's previous radiographs can yield valuable comparison information to the diagnostician. Such a requirement can prove beneficial to patients, health care personnel, and individuals concerned with quality control and proper practices in the health care setting.

\section{METHODS}

A one page anonymous survey was sent to licensed Florida Radiologists using randomization provided by Medical Marketing Services, Inc (MMS) (see Exhibit 1). The survey data was collected by Survey Monkey and stored at Nova Southeastern University. The number of radiologists registered within the MMS database in the state of Florida is 98. A number of surveys were also sent to radiologists working within the Memorial Healthcare System of Broward County, FL. The total number of responses collected was 22.

\section{RESULTS}

According to the collected data, fifty-nine percent of Florida radiologists never review mammograms during their practice. Twenty-three percent of radiologists review mammograms more than half the time, while eighteen percent review mammograms less than half the time (see Figure 1). Of the Florida radiologists who review mammograms, ninety-five percent believe that comparing past mammograms is very important in making a correct diagnosis. Five percent believe a comparison of films is somewhat important for a correct diagnosis (see Figure 2).

Moreover, seventy-two percent of Florida radiologists who read mammograms compare previous films more than half the time. However, twenty-two percent of those radiologists never compare past films, and six percent compare films less than half the time (see Figure 3). Twenty-four percent of Florida radiologists reading mammograms have been sued for malpractice at least one time (see Figure 4). There is a strong inverse relationship between comparing mammograms and likelihood to be sued. Florida radiologists who think comparing mammograms is "very important" were less likely to be sued for malpractice $(\mathrm{R}=0.99, \mathrm{p}<$ $0.0001)$. 


\section{Figure 1}

Exhibit 1 Physician Familiarity with Common Misdiagnoses

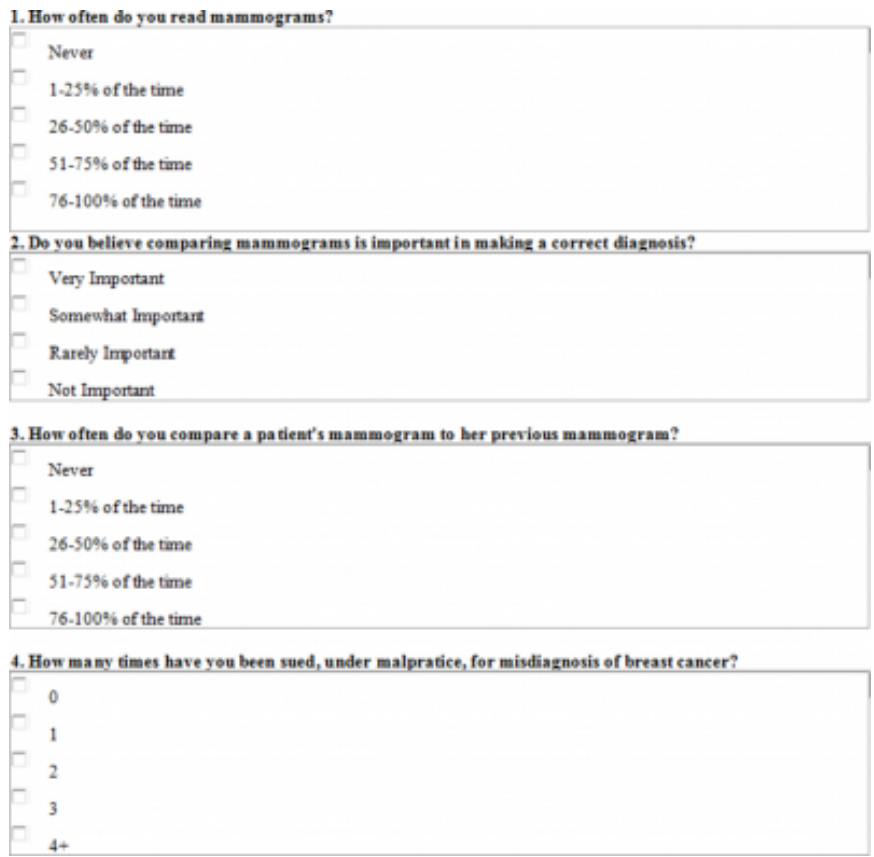

\section{Figure 2}

Figure 1

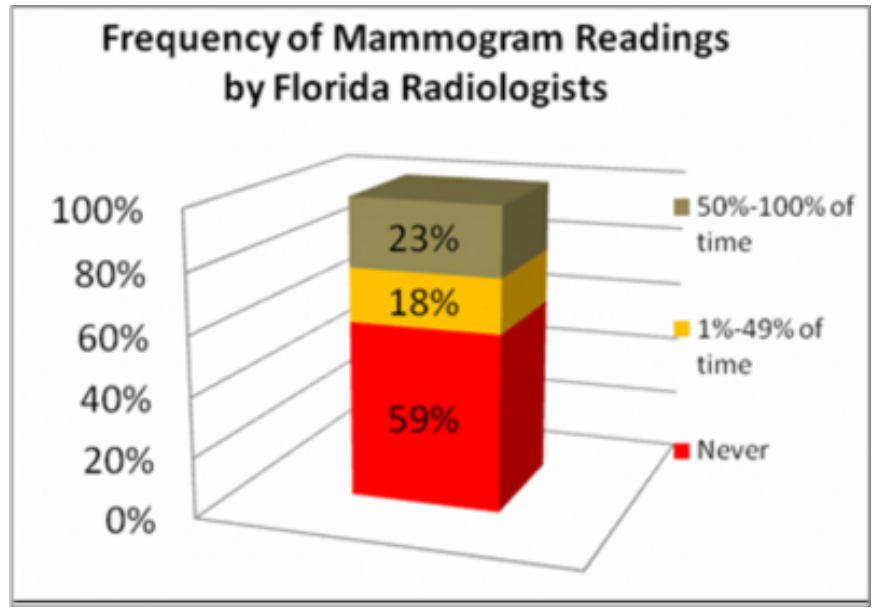

Figure 3

Figure 2

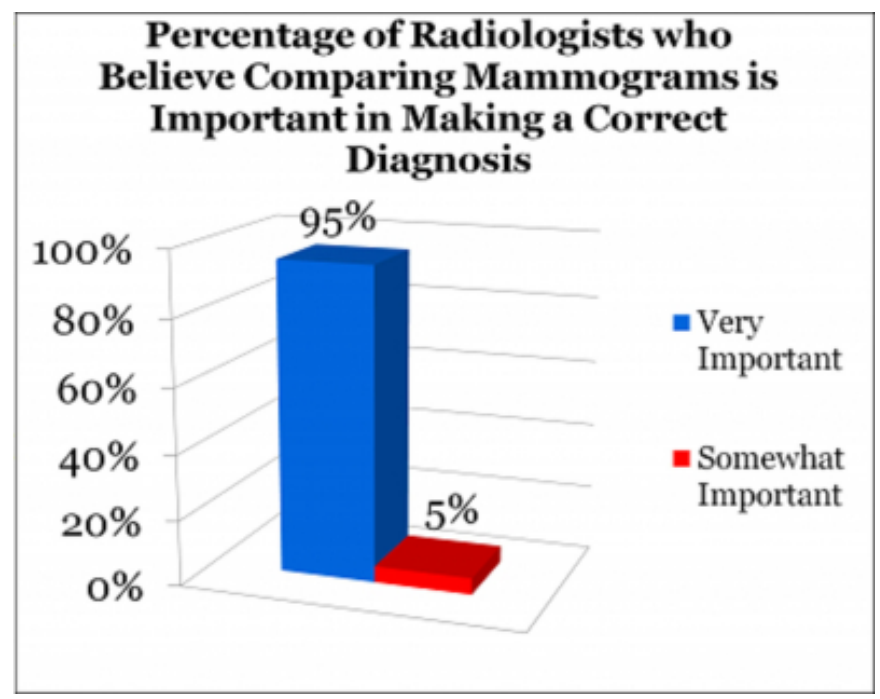

Figure 4

Figure 3

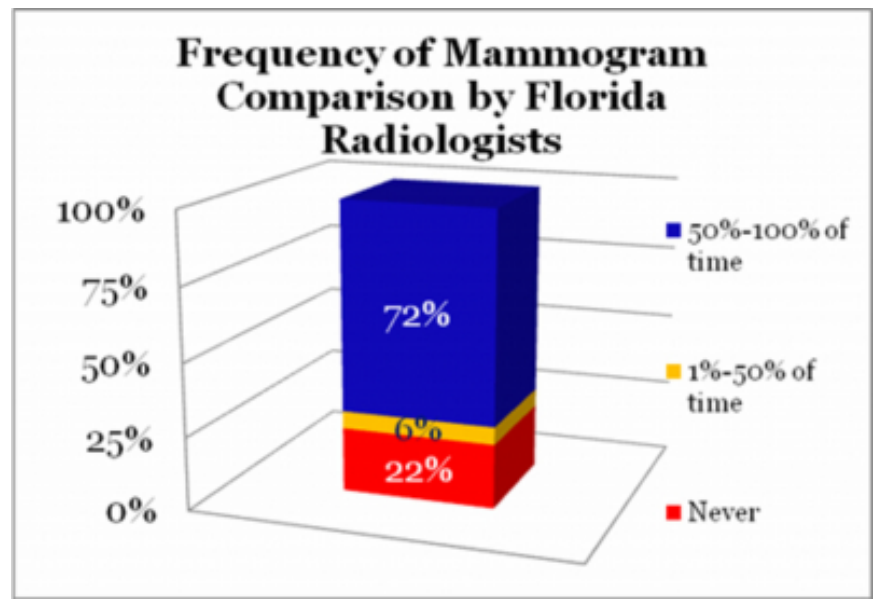

Figure 5

Figure 4

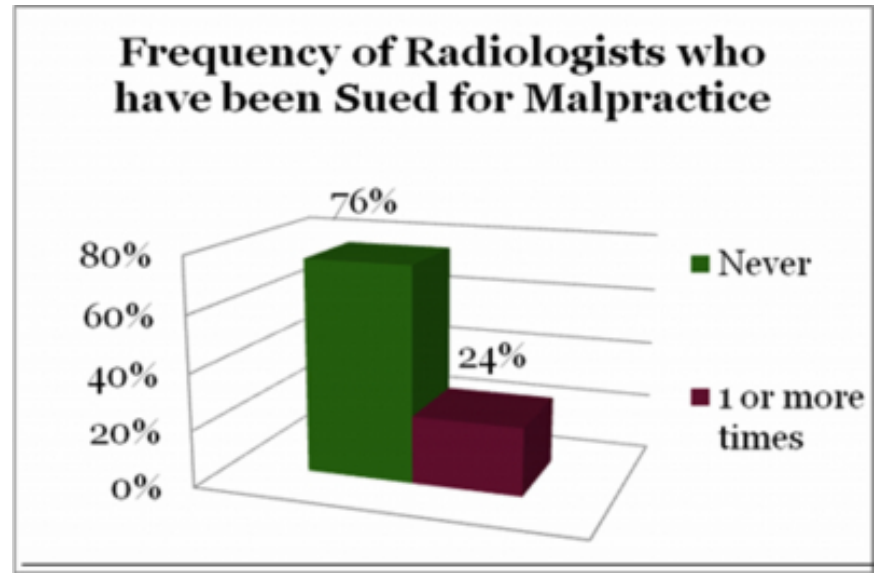




\section{DISCUSSION}

The objective of this study was to establish a relationship between mammogram comparison and the rates of failure to diagnosis breast cancer. The results show a strong inverse relationship — suggesting that an increase of mammogram comparison by radiologists will likely decrease the rates of misdiagnosis and the failure to diagnose breast cancer.

As the results show, even though $95 \%$ of Florida

Radiologists believe comparing mammograms is "very important" in making a correct diagnosis, only $72 \%$ of radiologists actually compare films more than half the time. It is interesting to note that of the population of radiologists who routinely compare past films, more than $75 \%$ have never been sued for malpractice. Of the population of radiologists that do not compare or track breast tissues from past mammograms, approximately $25 \%$ of them have been sued at least once for malpractice in the failure of diagnosing breast cancer. Thus, the authors of this study conclude that those radiologists who routinely compared and tracked past mammograms to current mammograms more often correctly diagnosed breast tissues, and as a result were less commonly sued for malpractice ( $\mathrm{R}=0.99, \mathrm{P}<0.0001)$. This conclusion is in agreement with previous studies, in which comparison was shown to be useful in establishing a correct diagnosis. It is anticipated that the practice of comparing mammograms with the previous exam will become a part of standard of care for reading mammograms by radiologists.

In order to determine why comparison mammography is not practiced by all radiologists, it is necessary to evaluate some of those factors which inhibit a radiologist from comparing previous mammograms. For example, closure of medical offices, misfiling/losing of radiographic films, added expense of duplicating copies of the films for patients, and among other reasons are just a variety of factors that hinder the radiologist from accurately diagnosing or screening suspicious breast tissues. In order to reduce misdiagnosis rates and the failure to diagnose breast cancer, all internists, family physicians, and physician sub-specialists must encourage women to find a convenient facility and to continue to have their annual mammograms conducted at the same facility, if possible. Also if there is a need to change the facility, patients should be educated to request a copy of their prior films from the previous centers. Moreover, patients should be encouraged to request digital copies of their radiographic films, such as on compact discs, for long term storage and for the ease of access to the films. Primary care physicians and radiologists must help to address these issues in order for mammogram comparisons to become more easily available.

With increased patient education and medical facility compliance, the rates of misdiagnosis may be reduced, along with patients receiving medical treatment earlier due to better detection rates. Since radiologists agree that comparing mammograms is essential, continuing medical education courses must mandate comparison as a standard of care, and mammogram comparison must become part of routine procedure.

\section{ACKNOWLEDGEMENTS}

This research was made possible by the generous support of Nova Southeastern University College of Osteopathic Medicine (NSU-COM).

\section{References}

1. Gandhi, T.K., Kachalia, A., Thomas, E.J., Puopolo, A.L., Yoon, C., Brennan, T.A., \& Studdert, D.M: Missed and delayed diagnoses in the ambulatory setting: a study of closed malpractice claims. Annals of Internal Medicine. 2006, 488-496.

2. Katherine Hobson: To Screen-or Not: Questions to put to your physician before having a test. U.S. News \& World Report, 70-74.

3. Szabo, Liz: U.S. Breast Cancer Rates Drop Dramatically. Retrieved December 31, 2008, Web site:

http://www.usatoday.com/news/health/2006-12-14-breast-ca ncer x.htm

4. SM Domchek, BL Weber: Clinical management of BRCA1 and BRCA2 mutation carriers. Oncogene, 25(43), 5825-31.

5. Sutter Health: Breast Cancer Project. Retrieved December 31, 2008, Web site:

http://www.sutterhealth.org/about/clinicalinit/ci_breast.html 6. Natasha K. Stout, Marjorie A. Rosenberg, Amy

Trentham-Dietz, Maureen A. Smith, Stephen M. Robinson,

Dennis G. Fryback: Retrospective Cost-effectiveness

Analysis of Screening Mammography. Journal of the

National Cancer Institute, 98(11), 774-782.

7. Sue M Moss, Howard Cuckle, Andy Evans, Louise Johns, et al: Effect of mammographic screening from age 40 years on breast cancer mortality at 10 years' follow-up: a randomised controlled trial. The Lancet, 368(9552), 2053-2060. 


\title{
Author Information
}

Nitin A. Wadhwa, BS

College of Osteopathic Medicine, Nova Southeastern University

\section{Marlow B. Hernandez, BS}

College of Osteopathic Medicine, Nova Southeastern University

\section{Debra C. Steinkohl, MHSA}

College of Osteopathic Medicine, Nova Southeastern University

\section{Anjali Bhasin, MD}

College of Osteopathic Medicine, Nova Southeastern University

\section{Chris Bailey, BS}

College of Osteopathic Medicine, Nova Southeastern University

\author{
Alina M. Perez, JD, MPH \\ Master of Public Health Program
}

